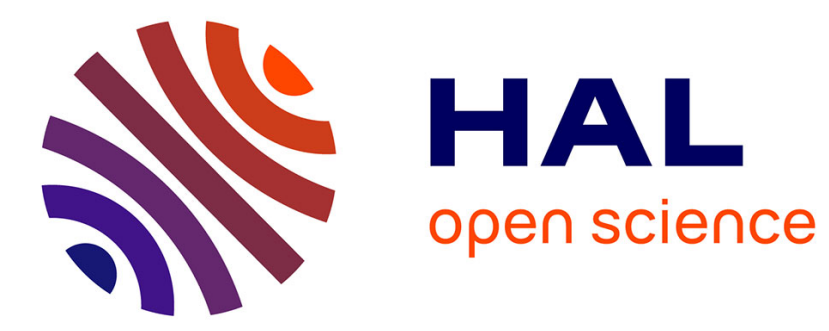

\title{
Detection of squeezed phonons in pump-probe spectroscopy
}

\author{
Massil Lakehal, Marco Schiro, Ilya M. Eremin, Indranil Paul
}

\section{To cite this version:}

Massil Lakehal, Marco Schiro, Ilya M. Eremin, Indranil Paul. Detection of squeezed phonons in pump-probe spectroscopy. Physical Review B, 2020, 10.1103/PhysRevB.102.174316 . hal-03059096

\section{HAL Id: hal-03059096 \\ https://hal.science/hal-03059096}

Submitted on 2 Nov 2021

HAL is a multi-disciplinary open access archive for the deposit and dissemination of scientific research documents, whether they are published or not. The documents may come from teaching and research institutions in France or abroad, or from public or private research centers.
L'archive ouverte pluridisciplinaire HAL, est destinée au dépôt et à la diffusion de documents scientifiques de niveau recherche, publiés ou non, émanant des établissements d'enseignement et de recherche français ou étrangers, des laboratoires publics ou privés. 


\title{
Detection of squeezed phonons in pump-probe spectroscopy
}

\author{
Massil Lakehal $\odot,{ }^{1}$ Marco Schiró,,${ }^{2, *}$ Ilya M. Eremin, ${ }^{3,4}$ and Indranil Paul ${ }^{\dagger}$ \\ ${ }^{1}$ Laboratoire Matériaux et Phénomènes Quantiques, Université de Paris, CNRS, Paris 75013, France \\ ${ }^{2}$ JEIP, USR 3573 CNRS, Collège de France, PSL Research University, F-75321 Paris, France \\ ${ }^{3}$ Institut für Theoretische Physik III, Ruhr-Universität Bochum, D-44801 Bochum, Germany \\ ${ }^{4}$ National University of Science and Technology MISiS, 119049 Moscow, Russian Federation
}

(Received 6 April 2020; accepted 10 November 2020; published 25 November 2020)

\begin{abstract}
Robust engineering of phonon squeezed states in optically excited solids has emerged as a promising tool to control and manipulate their properties. However, in contrast to quantum optical systems, detection of phonon squeezing is subtle and elusive, and an important question is what constitutes an unambiguous signature of it. The state of the art involves observing oscillations at twice the phonon frequency in time-resolved measurements of the out-of-equilibrium phonon fluctuation. Using the Keldysh formalism we show that such a signal is a necessary but not a sufficient signature of a squeezed phonon, since we identify several mechanisms that do not involve squeezing and yet produce similar oscillations. We show that reliable detection requires a time- and frequency-resolved measurement of the phonon spectral function.
\end{abstract}

DOI: 10.1103/PhysRevB.102.174316

\section{INTRODUCTION}

Recent advances in ultrafast pump-probe techniques have opened the possibility of controlling quantum materials by light [1-4]. This includes manipulating electronic orders [5-10], as well as controlling the lattice dynamics. Thus, it is well known that femtosecond pumping can create a coherent phonon, which manifests in the transient optical properties as oscillations with the phonon frequency $\omega_{0}$ [11-16], or induce changes to the electronic structure [17-20].

An intriguing newer goal is to engineer phonons into nonclassical states such as squeezed states [21,22] that can be described by a density matrix $\rho_{\mathrm{sq}} \equiv S^{\dagger} \rho_{\mathrm{th}} S$, where $S=\exp \left[\operatorname{ir}\left(b^{2}+\left(b^{\dagger}\right)^{2}\right)\right]$, with $\left(b, b^{\dagger}\right)$ the phonon annihilation/creation operators, $r$ the squeezing parameter, and $\rho_{\text {th }}$ the thermal density matrix. This can be promising for light-induced superconductivity $[23,24]$. Engineering of squeezed states is also a major goal in quantum technologies, with broad applications from metrology to quantum information $[25,26]$.

A related important goal is to unambiguously detect a squeezed state once it has been created. Several pump-probe experiments have reported a signature of phonon squeezing, based on measurements of the fluctuation $N_{X}(t) \equiv\left\langle X(t)^{2}\right\rangle-$ $\langle X(t)\rangle^{2}$ of the displacement $X$ of a phonon showing oscillations with frequency $2 \omega_{0}$ [27-35]. Indeed, theoretically, when a harmonic oscillator is squeezed impulsively at time $t=0$, it leads to $N_{X}(t) \sim \sin \left(2 \omega_{0} t\right)$ at subsequent times $t>0$. In contrast, since the average $\langle X(t)\rangle$ is subtracted in $N_{X}(t)$, per se, the fluctuations of coherent phonons do not have such

\footnotetext{
* On leave from Institut de Physique Théorique, Université Paris Saclay, CNRS, CEA, F-91191 Gif-sur-Yvette, France.

†indranil.paul@univ-paris-diderot.fr
}

oscillatory noise, unless interaction effects induce it via higher harmonic generation [see case (iii) below].

The purpose of the current work is twofold. (i) We bring into question the above state of the art that relates $2 \omega_{0}$ noise oscillation with squeezing. As we show below, there are out-of-equilibrium mechanisms that do not involve phonon squeezing and yet lead to $N_{X}(t) \sim \sin \left(2 \omega_{0} t\right)$. Thus, by itself, such a signal is not sufficient to conclude having a phonon squeezed state. In fact, the main ingredient for a $2 \omega_{0}$ oscillatory signal appears to be the breaking of time translation symmetry by a quench, which may or may not involve squeezing the phonon. (ii) We identify a more reliable method to detect a squeezed phonon state, which involves both time- and frequency-resolved Raman measurement.

\section{MODEL}

We consider a Brillouin-zone-center Raman-active optical phonon with dimensionless canonical variables $(X, P)$, coupled to a spinless fermionic bath with dispersion $\epsilon_{\mathbf{k}}$, with momentum $\mathbf{k}$, and described by operators $\left(c_{\mathbf{k}}^{\dagger}, c_{\mathbf{k}}\right)$ [see Fig. 1(a)]. The equilibrium Hamiltonian is

$$
\mathcal{H}=\frac{\hbar \omega_{0}}{2}\left(X^{2}+P^{2}\right)+\sum_{\mathbf{k}}\left(\epsilon_{\mathbf{k}}+\frac{V X}{\sqrt{2 N}} F_{\mathbf{k}}\right) c_{\mathbf{k}}^{\dagger} c_{\mathbf{k}} .
$$

$V$ is the phonon-bath interaction energy, assumed to be low enough that it can be treated perturbatively. $F_{\mathbf{k}}$ is a form factor that reflects the point-group symmetry of the phonon, and $N$ is the total number of bath variables. The role of the bath is simply to provide a finite inverse lifetime $\gamma$ to the phonon and to define a temperature $T$ for the system. We assume $\omega_{0}>\gamma$. The system is subjected to a laser pump pulse of femtosecond duration at time $t=0$, and we consider three possible outcomes of this out-of-equilibrium 


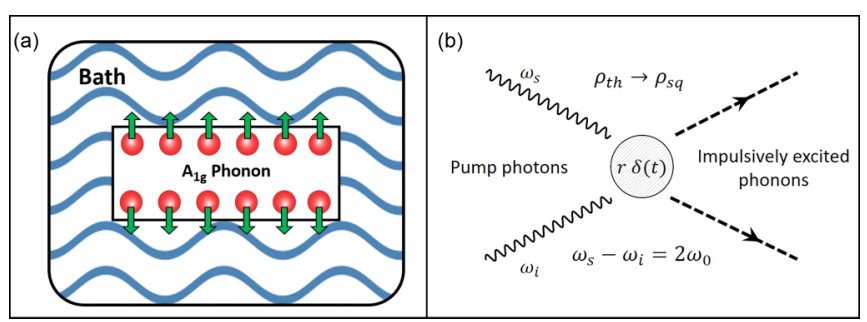

FIG. 1. (a) Sketch of the system: a Raman phonon, frequency $\omega_{0}$, in a bath. (b) A pump-induced impulsive second-order Raman process at time $t=0$ squeezing the phonon's thermal density matrix $\rho_{\text {th }} \rightarrow \rho_{\text {sq }}$, with $r$ the squeeze parameter. $\omega_{i}\left(\omega_{s}\right)$ is the incident (scattered) pump photon frequency.

perturbation that can manifest at a picosecond scale. (i) We consider the outcome that the phonon is squeezed by the pump via an impulsive resonant second-order Raman scattering process which leads to the generation/absorption of two phonon quanta [see Fig. 1(b)]. The perturbation is described by $\delta \mathcal{H}_{(i)}=r \delta(t)\left(X^{2}-P^{2}\right) / 2$, where $r$ is the dimensionless squeezing factor. (ii) We consider the outcome that the pump leads to heating of the bath, such that the bath temperature becomes time dependent. We model the thermal quench as $T(t)=T_{L}$ for $t<0$, and $T(t)=T_{L}+\left(T_{H}-T_{L}\right) e^{-t / \tau_{e}}$, with $T_{H} \geqslant T_{L}$ [see Fig. 2(a)]. Here $\tau_{e}$ is the thermal relaxation time scale of the bath. In this case the bath density matrix is perturbed. (iii) We consider the outcome that the phonon, assuming it has a symmetry-allowed cubic anharmonic potential, is coherently excited by the pump pulse [see Fig. 2(b)]. The generation mechanism of the coherent phonon, either via impulsive Raman scattering [15] or via displacive excitation [14], is unimportant here. This outcome is described by $\delta \mathcal{H}_{(i i i)}=\hbar \omega_{0} f(t) X+\lambda X^{3} / 3$, where $f(t)$ is the time-dependent force that excites the phonon coherently, and $\lambda$ is the energy scale of the cubic anharmonic potential. Importantly, such a cubic anharmonicity is relevant in most systems that have been studied to date in the context of squeezing. These include fully symmetric $A_{g}$ phonons in all systems, as well as $E_{g}$ phonons in bismuth and $\alpha$-quartz with
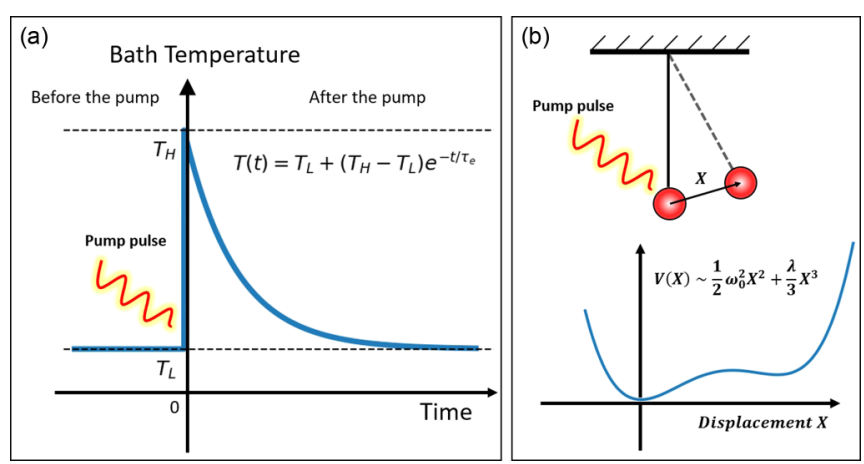

FIG. 2. Two out-of-equilibrium processes that do not involve creating a squeezed phonon with density matrix $\rho_{\mathrm{sq}}$, but whose noise signal $N_{X}(t) \sim \sin \left(2 \omega_{0} t\right)$ has the same oscillatory component as in the squeezed case. (a) The bath temperature is quenched by the pump. (b) A phonon, with a cubic anharmonic potential, is excited coherently by the pump. a $D_{3}$ point group. Note that for a low enough pump fluence, the signatures of all three of these outcomes depend linearly on the fluence.

Our goal is to study the fluctuation/noise $N_{X}(t)$ generated for each of the above three outcomes separately. We work using the Keldysh formalism, where the phonon coordinate is defined on a two-branch real-time contour, $X_{ \pm}(t)$. We integrate out the bath variables, treating the coupling $V$ to second order, and write the phonon action in the more physical classical and quantum basis $X_{\mathrm{cl} / q}=\left(X_{+} \pm X_{-}\right) / \sqrt{2}$ as [36,37]

$$
S[X]=\int_{-\infty}^{\infty} d t^{\prime} \int_{-\infty}^{\infty} d t X^{T}\left(t^{\prime}\right) \hat{D}_{\text {inv }}\left(t^{\prime}, t\right) X(t),
$$

where $X^{T}(t) \equiv\left(X_{\mathrm{cl}}(t), X_{q}(t)\right)$, and the matrix

$$
\hat{D}_{\text {inv }}\left(t^{\prime}, t\right) \equiv\left[\begin{array}{cc}
0 & D_{A}^{-1}\left(t^{\prime}, t\right) \\
D_{R}^{-1}\left(t^{\prime}, t\right) & -\Pi_{K}\left(t^{\prime}, t\right)
\end{array}\right] .
$$

$D_{R / A}^{-1}\left(t^{\prime}, t\right)=D_{0, R / A}^{-1}\left(t^{\prime}, t\right)-\Pi_{R / A}\left(t^{\prime}, t\right)$ are the inverse retarded (advanced) phonon propagators and $D_{0, R / A}^{-1}\left(t^{\prime}, t\right)$ are those of a free phonon. The self-energy contributions $\Pi_{R / A / K}\left(t^{\prime}, t\right)$ from the fermionic bath are given by

$$
\Pi_{R}\left(t^{\prime}, t\right)=\Pi_{\mathrm{cl}, q}\left(t^{\prime}, t\right) \equiv-i V^{2} \sum_{\mathbf{k}} F_{\mathbf{k}}^{2}\left\langle n_{\mathbf{k}, \mathrm{cl}}\left(t^{\prime}\right) n_{\mathbf{k}, q}(t)\right\rangle_{S_{b}^{0}},
$$

$\Pi_{A}\left(t^{\prime}, t\right)=\Pi_{q, \mathrm{cl}}\left(t^{\prime}, t\right)$, and $\Pi_{K}\left(t^{\prime}, t\right)=\Pi_{\mathrm{cl}, \mathrm{cl}}\left(t^{\prime}, t\right) . \quad S_{b}^{0}$ is the action of the bare bath, and $n_{\mathbf{k}, \mathrm{cl} / q} \equiv\left(c_{\mathbf{k}, \mathrm{cl}}^{\dagger} c_{\mathbf{k}, \mathrm{cl} / q}+\right.$ $\left.c_{\mathbf{k}, q}^{\dagger} c_{\mathbf{k}, q / \mathrm{cl}}\right) / \sqrt{2}$. From Eq. (2) we get

$$
\left\langle X_{\mathrm{cl}}(t)^{2}\right\rangle_{S[X]}=i \int_{-\infty}^{\infty} d t^{\prime \prime} d t^{\prime} D_{R}\left(t, t^{\prime \prime}\right) D_{R}\left(t, t^{\prime}\right) \Pi_{K}\left(t^{\prime \prime}, t^{\prime}\right),
$$

which is the out-of-equilibrium generalization of the fluctuation-dissipation relation. Our task is to obtain $D_{R}\left(t^{\prime}, t\right)$ and $\Pi_{K}\left(t^{\prime}, t\right)$ and, from these, to calculate the noise $N_{X}(t)$ for the above three cases.

\section{RESULTS}

Our main results are as follows. (1) We show that for all three cases the noise signal has a $2 \omega_{0}$ oscillatory component with $N_{X}(t) \sim \sin \left(2 \omega_{0} t\right)$. Yet, only in case (i) is the signal from a phonon squeezed state. Thus, a $2 \omega_{0}$ oscillatory noise signal is not sufficient to conclude whether or not the phonon is in the squeezed state $\rho_{\text {sq }}$. (2) By comparing the phonon spectral function for the three cases, we obtain a reliable method to detect a squeezed phonon state $\rho_{\mathrm{sq}}$ which involves time- and frequency-resolved Raman measurement.

\section{A. Case (i): Noise from squeezing}

As the bath itself is at equilibrium with temperature $T$, $\Pi_{K}\left(t^{\prime}, t\right)$ is a function of $\left(t^{\prime}-t\right)$, and its Fourier transform $\Pi_{K, \text { eq }}(\omega)$ satisfies standard fluctuation-dissipation theorem $\Pi_{K, \text { eq }}(\omega)=\left[\Pi_{R, \text { eq }}(\omega)-\Pi_{A, \text { eq }}(\omega)\right] \operatorname{coth}[\omega /(2 T)]$. As we are interested in the long-time dynamics of the phonon, it is enough to expand $\Pi_{R, \text { eq }}(\omega)$ in frequency. Since charge excitations are gapless in a good fermionic bath, we get $\operatorname{Im} \Pi_{R \text {,eq }}(\omega) \approx-\omega \gamma / \omega_{0}$ and $\Pi_{K \text {,eq }}(\omega) \approx$ $-2 i \omega \gamma \operatorname{coth}[\omega /(2 T)] / \omega_{0}$. Next, due to $\delta \mathcal{H}_{(i)}$ the squeezing 
perturbation, $D_{R}\left(t^{\prime}, t\right)$, satisfies

$$
\begin{aligned}
& {\left[h(t) \partial_{t}^{2}+\dot{h}(t) \partial_{t}+2 \gamma \partial_{t}+r \omega_{0} \delta(t)+\omega_{0}^{2}\right] D_{R}\left(t^{\prime}, t\right)} \\
& \quad=-2 \omega_{0} \delta\left(t^{\prime}-t\right)
\end{aligned}
$$

where $h(t) \equiv \omega_{0} /\left[\omega_{0}-r \delta(t)\right]$ and $\dot{h}(t)=\partial_{t} h(t)$, while $\gamma$ plays the role of phonon damping induced by the fermionic bath. The solution of the above equation with the initial conditions $D_{R}\left(t^{+}, t\right)=0$ and $\partial_{t} D_{R}\left(t^{+}, t\right)=-2 \omega_{0}$ is described in detail in Appendix A. Here we simply quote the final answer. Before that, for convenience, we introduce the notation

$$
\mathcal{F}_{T}\left[f\left(x_{1}, x_{2}, \ldots\right) ; x_{i} ; k_{i}\right] \equiv \int_{-\infty}^{\infty} d x_{i} e^{i k_{i} x_{i}} f\left(x_{1}, x_{2}, \ldots\right) .
$$

In terms of $\Delta_{R}(t, \omega) \equiv \mathcal{F}_{T}\left[D_{R}(t, t-\tau) ; \tau ; \omega\right]$ we get

$$
\Delta_{R}(t, \omega)=\left[1+\frac{i}{2 \omega_{0}} \theta(t) K(t, \omega) e^{i \omega t-\gamma t}\right] D_{R, \mathrm{eq}}(\omega),
$$

where $D_{R, \text { eq }}(\omega) \equiv 2 \omega_{0} /\left[\omega^{2}+2 i \gamma \omega-\omega_{0}^{2}\right]$ is the equilibrium propagator, and $K(t, \omega) \equiv A(t)\left(\omega-\omega_{0}+\right.$ $i \gamma) e^{i \omega_{0} t}+\bar{A}(t)\left(\omega+\omega_{0}+i \gamma\right) e^{-i \omega_{0} t}$. The function $A(t) \equiv$ $\sinh r \cos \left(2 \omega_{0} t\right)+i\left[\cosh r-\sinh r \sin \left(2 \omega_{0} t\right)-1\right] \quad$ has information about squeezing. Reexpressing Eq. (3) in terms of $\Delta_{R}(t, \omega)$ and $\Pi_{K, \text { eq }}(\omega)$ we get (see Appendix A)

$$
N_{X}(t)=\frac{1}{2} e^{-2 \gamma t} \sinh 2 r\left[\tanh r-\sin \left(2 \omega_{0} t\right)\right] \operatorname{coth} \frac{\omega_{0}}{2 T} .
$$

Thus, $N_{X}(t)$ has a $2 \omega_{0}$ oscillatory component.

\section{B. Case (ii): Noise from thermal quench of the bath}

Here we assume that the effect of the pump on the bath can be encoded by a slowly varying effective temperature, $T(s)=T_{L}+\theta(s)\left(T_{H}-T_{L}\right) e^{-s / \tau_{e}}$, for time scales much longer than those relevant for the internal electronic dynamics. Thus, we disregard the processes by which the bath itself thermalizes to an effective time-dependent temperature, and we study the consequences of such a pseudoequilibrium environment on the phonon noise.

The first effect of the pseudoequilibrium is that the Keldysh self-energy $\Pi_{K}\left(t^{\prime}, t\right)$, sensitive to the occupation of the bath modes, loses time-translational invariance and acquires a dependence on the average time $s \equiv\left(t^{\prime}+t\right) / 2$ via the temporally varying temperature. It is convenient to define $\Pi_{K}(s, \omega) \equiv \mathcal{F}_{T}\left[\Pi_{K}(s+\tau / 2, s-\right.$ $\tau / 2) ; \tau ; \omega]$. We expect that $\Pi_{K}(s, \omega)$, in analogy with $\Pi_{K \text {,eq }}(\omega)$, satisfies the fluctuation-dissipation relation with the time-dependent temperature $T(s)$. This implies that $\Pi_{K}(s, \omega) \approx-2 i \omega \gamma \operatorname{coth}[\omega /(2 T(s))] / \omega_{0}$. In principle, the retarded phonon propagator $D_{R}\left(t^{\prime}, t\right)$ also acquires $s$ dependence through the temperature dependence of damping $\gamma(s)$. However, to leading order in the temperature quench $\left(T_{H}-\right.$ $T_{L}$ ) this slow variation can be ignored, and one can use the equilibrium form $D_{R, \text { eq }}(\omega)$. Note that the above simplification does not affect the final conclusion qualitatively.

From the above considerations, it is simple to evaluate the fluctuations in terms of $\left\langle X_{\mathrm{cl}}(\Omega)^{2}\right\rangle_{S[X]} \equiv \mathcal{F}_{T}\left[\left\langle X_{\mathrm{cl}}(t)^{2}\right\rangle_{S[X]} ; t ; \Omega\right]$ from the relation

$$
\begin{aligned}
\left\langle X_{\mathrm{cl}}(\Omega)^{2}\right\rangle_{S[X]}= & \frac{i}{4 \pi} \int_{-\infty}^{\infty} d s \int_{-\infty}^{\infty} d \omega D_{R, \mathrm{eq}}(\omega+\Omega / 2) \\
& \times D_{R, \mathrm{eq}}^{*}(\omega-\Omega / 2) \Pi_{K}(s, \omega) e^{i \Omega s} .
\end{aligned}
$$

We assume that $\gamma$ is the lowest energy scale and, in particular, $\gamma \ll T_{L}$. Then

$$
\left\langle X_{\mathrm{cl}}\left(\Omega \sim \pm 2 \omega_{0}\right)^{2}\right\rangle_{S[X]} \propto \frac{1}{z_{1} z_{2}} \int_{-\infty}^{\infty} d s e^{i \Omega s} \Pi_{K}(s, \omega \rightarrow 0),
$$

where $z_{1,2} \equiv \Omega / 2 \pm \omega_{0}+i \gamma$. This implies that $\left\langle X_{\mathrm{cl}}(\Omega)^{2}\right\rangle_{S[X]}$ has poles at $\Omega= \pm 2 \omega_{0}$, which in the time domain translate as $\sin \left(2 \omega_{0} t\right)$ oscillations. Note that the origin of these poles is an equilibrium property of a damped oscillator. However, at equilibrium time translation symmetry ensures that $\Pi_{K}(s, \omega)$ does not depend upon the average time $s$. In this case the $s$ integral above gives $\delta(\Omega)$, and therefore, at equilibrium the finite- $\Omega$ poles are "inaccessible." Keeping the $s$ dependence of $\Pi_{K}(s, \omega)$ we get

$$
N_{X}(t)=\frac{-\gamma\left(T_{H}-T_{L}\right)}{2 \omega_{0}^{2}} e^{-2 \gamma t} \sin \left(2 \omega_{0} t\right)+\ldots,
$$

where the ellipsis implies nonoscillatory contributions. Thus, we demonstrate that $N_{X}(t)$ can have a $2 \omega_{0}$ oscillatory signal simply due to pump-induced thermal quench of the bath. In fact, any out-of-equilibrium perturbation of the bath that breaks time translation symmetry will lead to a $2 \omega_{0}$ oscillatory noise signal.

\section{Case (iii): Noise from phonons with cubic anharmonicity coherently excited}

This is relevant for $A_{g}$ phonons in all systems and, also, for $E_{g}$ phonons in $D_{3}$ symmetric systems such as $\alpha$-quartz and bismuth. The pump leads to $\left\langle X_{\mathrm{cl}}(t)\right\rangle_{S[X]} \equiv u(t) \neq 0$, where $u(t)$ describes the coherent excitation. To study the fluctuations around the average we expand the action in terms of $\delta X \equiv X-u(t)$. This gives Eq. (2), with $X_{\mathrm{cl}}$ replaced by $\delta X_{\mathrm{cl}}$ and with the propagator $D_{R}\left(t^{\prime}, t\right)$ satisfying the equation

$$
\left[\partial_{t}^{2}+2 \gamma \partial_{t}+\omega_{0}^{2}+4 \lambda \omega_{0} u(t)\right] D_{R}\left(t^{\prime}, t\right)=-2 \omega_{0} \delta\left(t^{\prime}-t\right) .
$$

In the above the crucial ingredient is the $\lambda u(t)$ term, which acts as a time-dependent potential for the fluctuations. We assume $\lambda / \omega_{0} \ll 1$, such that it is sufficient to solve the above equation to linear order in $\lambda$. We expand $D_{R}\left(t^{\prime}, t\right)=$ $D_{R, \text { eq }}\left(t^{\prime}-t\right)+\lambda D_{R, 1}\left(t^{\prime}, t\right)$ with

$$
D_{R, 1}\left(t^{\prime}, t\right)=2 \int_{-\infty}^{\infty} d s D_{R, \text { eq }}\left(t^{\prime}-s\right) u(s) D_{R, \text { eq }}(s-t) .
$$

On the other hand, the bath is at equilibrium with temperature $T$, and therefore the Keldysh self-energy is the one at equilibrium $\Pi_{K, \text { eq }}(\omega)$. Ignoring the equilibrium contribution to noise we get

$$
\begin{aligned}
& N_{X}(t)_{\text {noneq }} \\
& \quad=-i \lambda \int_{-\infty}^{\infty} d s d s^{\prime} D_{R, \text { eq }}(t-s) D_{R, 1}\left(t, s^{\prime}\right) \Pi_{K, \text { eq }}\left(s-s^{\prime}\right) \\
& =4 \lambda \operatorname{coth}\left(\frac{\omega_{0}}{2 T}\right) \int_{0}^{t} d s u(s) \sin \left[2 \omega_{0}(t-s)\right] e^{-2 \gamma(t-s)} .
\end{aligned}
$$

In the last line the $s$ integral starts from $s>0$ because the coherent excitation is triggered by the pump at $s=0$. This element of time translation symmetry breaking is crucial. Irrespective of the details of the coherent motion $u(s)$, it is 
clear from the above that there is an oscillatory $2 \omega_{0}$ signal in the noise. The precise form of $N_{X}(t)$ depends on the force $f(s)$, which determines $u(s)$. An impulsive excitation leads to $u(s)=u_{0} \sin \left(\omega_{0} s\right) e^{-\gamma s}$, and

$$
N_{X}(t)_{\text {noneq }}=-\frac{4 \lambda u_{0} \operatorname{coth}\left[\omega_{0} /(2 T)\right]}{3 \omega_{0}} e^{-2 \gamma t} \sin \left(2 \omega_{0} t\right)+\ldots,
$$

where the ellipsis implies terms not relevant for the current discussion. For a displacive coherent excitation the sine signal is replaced by a cosine, which reflects the property of coherent phonons that the phase of the oscillation is determined by the generation mechanism [14-16]. Thus, as in case (ii), we obtain an oscillatory $2 \omega_{0}$ noise signal without having created the density matrix $\rho_{s q}$. However, in contrast to case (ii), which involves only incoherent excitations, in (iii) the $2 \omega_{0}$ signal is built out of a coherent excitation at $\omega_{0}$. Consequently, the latter is analogous to higher harmonic generation.

\section{Reliable signature of a squeezed phonon}

Next, we identify a measurable quantity that can distinguish a squeezed phonon, i.e., case (i) from cases (ii) and (iii). Since the noise $N_{X}(t)$, which is a time-resolved but frequencyintegrated quantity, has the same oscillatory property for the three situations, a promising direction is to look for a quantity which is both time and frequency resolved. With this intuition we define the time- and frequency-resolved spectral function

$$
A(t, \omega) \equiv \operatorname{Im} D_{R}(t, \omega),
$$

where $D_{R}(t, \omega) \equiv \mathcal{F}_{T}\left[D_{R}(t+\tau / 2, t-\tau / 2) ; \tau ; \omega\right]$ is the Wigner transform of the two-time retarded propagator. Note that $D_{R}(t, \omega)$ is to be distinguished from $\Delta_{R}(t, \omega)$ defined earlier. At equilibrium, $A(t, \omega)$ is $t$ independent and is peaked at $\omega= \pm \omega_{0}$. Thus, $A\left(t, \omega_{0}\right)$ describes how the spectral peak varies with time $t$ after the pump.

We find that, indeed, the $t$ dependencies of $A\left(t, \omega_{0}\right)$ for the three cases are distinct, since the phonon propagators $D_{R}(t, \omega)$ in the three cases are quite different (for details see Appendix B). In case (i) squeezing leads $A\left(t, \omega_{0}\right)$ to oscillate at frequency $4 \omega_{0}$ as a function of $t$ for the following reason. Since squeezing involves excitation of two phonon quanta, $D_{R}(t+\tau / 2, t-\tau / 2) \sim \cos \left(2 \omega_{0} t\right) \theta(\tau-2 t)$. The constraint $\tau \geqslant 2 t$ is crucial and follows from the fact that squeezing is an impulsive process occurring at time 0 [see Fig. 1(b)], and so the two time arguments in $D_{R}(t+\tau / 2, t-\tau / 2)$ must have opposite signs [see Eq. (A19) in Appendix A]. Next, the Wigner transform involves multiplication of the phase $\exp (i \omega \tau)$, and therefore, imposing the constraint leads, via phase accumulation, to $A\left(t, \omega_{0}\right) \sim \cos \left(4 \omega_{0} t+\phi\right)$, where $\phi$ is a phase that depends on details. Thus, the $4 \omega_{0}$ oscillation is a consequence of impulsive excitation of two phonon quanta, which is the hallmark of squeezing. In case (ii) $A\left(t, \omega_{0}\right)$ is practically $t$ independent, since the weak $t$ dependence of the phonon self-energy induced by the electronic bath can be disregarded. In case (iii) $A\left(t, \omega_{0}\right)$ has oscillations at frequencies $4 \omega_{0}$, but also at $\omega_{0}$. The latter is due to the fact that in this scenario the phonon is excited coherently. This exemplifies that processes involving higher harmonic generation invariably will have a signature at a frequency lower than $4 \omega_{0}$, and thus, they can be distinguished from squeezing.

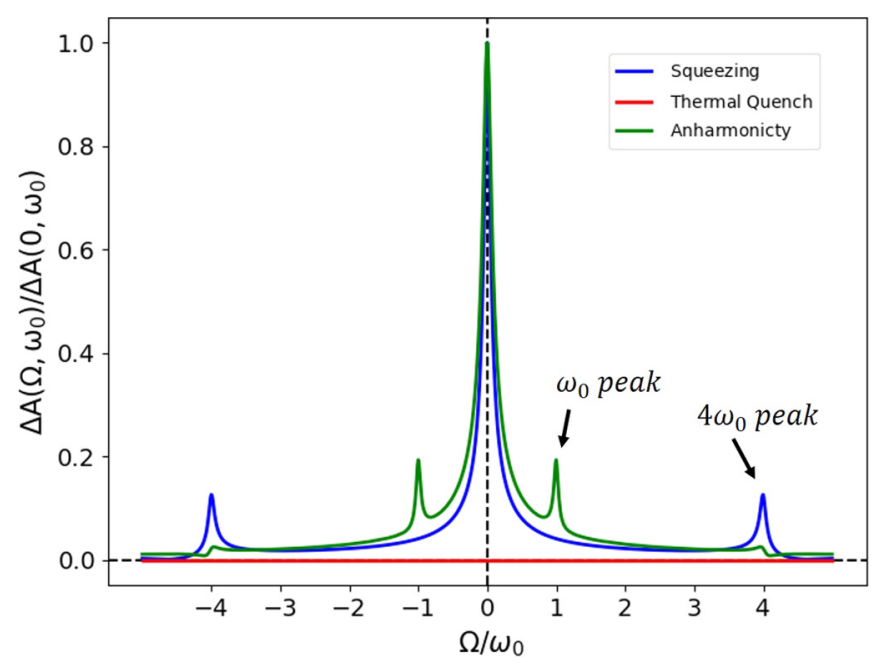

FIG. 3. The time dependence of the spectral function peak $\Delta A\left(t, \omega_{0}\right) \equiv A\left(t, \omega_{0}\right)-A\left(0, \omega_{0}\right)$ [see Eq. (8) for definition] and its Fourier transform $\Delta A\left(\Omega, \omega_{0}\right)$ have distinct signatures for the three cases studied here. A distinguishing feature of squeezing is a single peak in $\left|\Delta A\left(\Omega, \omega_{0}\right)\right|$ at $\Omega=4 \omega_{0}$.

These three distinct $t$ dependencies can be conveniently expressed in terms of $\Delta A\left(\Omega, \omega_{0}\right) \equiv \mathcal{F}_{T}\left[\Delta A\left(t, \omega_{0}\right) ; t ; \Omega\right]$, where $\Delta A\left(t, \omega_{0}\right) \equiv A\left(t, \omega_{0}\right)-A\left(0, \omega_{0}\right)$ is the time-dependent part of the spectral function peak. Thus, as shown in Fig. 3, a distinguishing feature of squeezing is a single peak in $\left|\Delta A\left(\Omega, \omega_{0}\right)\right|$ at $\Omega=4 \omega_{0}$.

Finally, we discuss how the time-dependent spectral function $A(t, \omega)$ can be measured. At equilibrium this is standard since the Raman scattering cross section is proportional to the correlation function, from which the spectral function can be deduced using the fluctuation-dissipation relation. The issue is nontrivial in an out-of-equilibrium situation since the standard fluctuation-dissipation relation is not valid. However, we can generalize the relation in the following manner. According to the theory of time- and frequency-resolved Raman spectroscopy the scattering intensity $I(t, \omega) \propto D^{>}(t, \omega)$, where $D^{>}(t, \omega)$ is the Wigner transform of the two times greater function [38,39]. Then, from standard Keldysh theory it follows that $I(t, \omega)-I(t,-\omega) \propto A(t, \omega)$ (see Appendix C). In other words, the time-dependent spectral function can be extracted from the difference in the Stokes and anti-Stokes Raman intensities. In practice, the probe cycle will require two pulses, one which is time resolved and a second which is frequency resolved [40,41].

\section{CONCLUSION}

To summarize, we studied the fluctuations of a phonon coupled to a bath in a pump-probe setup. We argued that the noise or the variance associated with the atomic displacement oscillates at twice the phonon frequency $\omega_{0}$, i.e., $N_{X}(t) \equiv$ $\left\langle X(t)^{2}\right\rangle-\langle X(t)\rangle^{2} \sim \sin \left(2 \omega_{0} t\right)$, due to pump-induced breaking of time translation symmetry. Thus, such oscillations cannot be taken as proof of having prepared the phonon in a squeezed state with density matrix $\rho_{\text {sq }}$. A reliable way to identify squeezed phonons involves a study of the time-dependent 
spectral function of the phonon, which can be achieved by a time- and frequency-resolved Raman measurement.

\section{ACKNOWLEDGMENTS}

We are thankful to A. Le Boité, R. Chitra, M. Eckstein, D. Fausti, S. Florens, Y. Gallais, S. Johnson, and Y. Laplace for insightful discussions. M.L. and I.P. acknowledge financial support from ANR-DFG Grant No. ANR-15-CE30-0025. I.M.E. was supported by the joint DFG-ANR Project (ER 463/8-1). He also acknowledges partial support from the Ministry of Science and Higher Education of the Russian Federation in the framework of Increase Competitiveness Program of NUST MISiS Grant No. K2-2020-038.

\section{APPENDIX A: RETARDED GREEN'S FUNCTION OF A SQUEEZED PHONON}

The retarded phonon Green's function of a squeezed phonon coupled to a thermal bath satisfies the equation of motion

$$
\begin{aligned}
& \frac{1}{2 \omega_{0}}\left(h(t) \partial_{t}^{2}+\dot{h}(t) \partial_{t}+2 \gamma \partial_{t}+\omega_{0}^{2}+g(t) \omega_{0}\right) D_{R}\left(t, t^{\prime}\right) \\
& =\delta\left(t-t^{\prime}\right)
\end{aligned}
$$

where $h(t)=\omega_{0} /\left(\omega_{0}-r g(t)\right)$, and $g(t)$ describes the envelope of the pump pulse. We assume that the width of the pump pulse is the smallest time scale of the problem and, hence, that it can be well approximated by a Dirac distribution. It is, however, uncomfortable to deal with the nonanalyticity of the Dirac distribution in path integrals. Indeed, for a delta-shaped pump pulse centered at time $t=0$, the displacement $X_{\mathrm{cl} / q}(t)$ and momentum $P_{\mathrm{cl} / q}(t)(t)$ fields are not well defined at initial time $t=0$. In practice, however, the shape of a physical pump pulse is a smooth function with a finite width $\tau_{p}$, and the approximation with a Dirac distribution is just a practical mathematical description. Therefore, to avoid complication, we solve the problem for a general pulse with a smooth envelope $g(t)$ centered at time $t=0$ and a finite width $\tau_{p}$ and only take the limit $\tau_{p} \rightarrow 0$ for which $g(t) \rightarrow r \delta(t)$ at the end of the calculation.

The Dirac delta function $\delta\left(t-t^{\prime}\right)$ is vanishing for $t \neq t^{\prime}$, hence, for time $t \neq t^{\prime}$ the Green's function satisfies the homogeneous equation

$$
\left(h(t) \partial_{t}^{2}+h^{\prime}(t) \partial_{t}+2 \gamma \partial_{t}+\omega_{0}^{2}+g(t) \omega_{0}\right) D_{R}\left(t, t^{\prime}\right)=0 .
$$

The retarded Green's function has a causal structure, i.e., it vanishes for $t<t^{\prime}$, and satisfies a second-order differential equation whose boundary conditions are given by the definition of the retarded Green's function at equal time,

$$
D_{R}\left(t^{+}, t\right)=-i\left\langle\left[\hat{X}\left(t^{+}\right), \hat{X}(t)\right]\right\rangle=0,
$$

and the jump condition imposed by the delta function,

$$
\partial_{t} D_{R}\left(t^{+}, t\right)=-2 \omega_{0},
$$

where $t^{+}=t+0^{+}$.
We replace the equation of the retarded Green's function, Eq. (A1), by a set of two coupled first-order equations and write

$$
\begin{aligned}
\partial_{t} D_{R}(t, s) & =\left[\omega_{0}-g(t)\right] K(t, s), \\
\partial_{t} K(t, s) & =-\left[\omega_{0}+g(t)\right] D_{R}(t, s)-2 \frac{\gamma}{\omega_{0}} \partial_{t} D_{R}(t, s),
\end{aligned}
$$

where $K(t, s)$ is a function that we introduce as a mathematical tool. Note that for $g(t)=0$, the equation of the retarded Green's function, Eq. (A5), is that of a damped harmonic oscillator. Therefore, we propose the following ansatz for the solution:

$$
\begin{aligned}
D_{R}(t, s)= & X_{0}(t, s) \cos (\Omega t) e^{-\gamma t} \\
& +\frac{1}{\Omega}\left[\omega_{0} P_{0}(t, s)+\gamma X_{0}(t, s)\right] \sin (\Omega t) e^{-\gamma t}, \\
K(t, s)= & P_{0}(t, s) \cos (\Omega t) e^{-\gamma t} \\
& -\frac{1}{\Omega}\left[\omega_{0} X_{0}(t, s)+\gamma P_{0}(t, s)\right] \sin (\Omega t) e^{-\gamma t},
\end{aligned}
$$

where $\Omega^{2}=\omega_{0}^{2}-\gamma^{2}$. This form of the solution ensures that for constant $X_{0}(t, s)$ and $P_{0}(t, s)$, the functions $D_{R}(t, s)$ and $K(t, s)$ satisfy the equation of motion of a damped harmonic oscillator. The fact that the phonon is a well-defined excitation implies that $\gamma \ll \omega_{0}$. Thus, we write the equations of motion for $X_{0}(t, s)$ and $P_{0}(t, s)$ in this limit and find that

$$
\left[\begin{array}{l}
\partial_{t} X_{0}(t, s) \\
\partial_{t} P_{0}(t, s)
\end{array}\right]=-g(t)\left[\begin{array}{cc}
-\sin (2 \Omega t) & \cos (2 \Omega t) \\
\cos (2 \Omega t) & \sin (2 \Omega t)
\end{array}\right]\left[\begin{array}{l}
X_{0}(t, s) \\
P_{0}(t, s)
\end{array}\right] .
$$

We take the limit where the width of the pump pulse goes to $0, \tau_{p} \rightarrow 0$, and recover the Dirac delta function, $g(t) \rightarrow r \delta(t)$. Using the properties of the Dirac distribution, we have that

$$
\begin{aligned}
& \sin (\Omega t) g(t)=\sin (0) g(t)=0, \\
& \cos (\Omega t) g(t)=\cos (0) g(t)=g(t),
\end{aligned}
$$

hence Eq. (A7) further simplifies, and we obtain

$$
\left[\begin{array}{l}
\partial_{t} X_{0}(t, s) \\
\partial_{t} P_{0}(t, s)
\end{array}\right]=-g(t)\left[\begin{array}{ll}
0 & 1 \\
1 & 0
\end{array}\right]\left[\begin{array}{l}
X_{0}(t, s) \\
P_{0}(t, s)
\end{array}\right] .
$$

The solution of this equation of motion is straightforward, and after some algebraic manipulations we find that

$$
\begin{aligned}
X_{0}(t, s) & =\operatorname{ch}[F(t, s)] K_{1}(s)-\operatorname{sh}[F(t, s)] K_{2}(s), \\
P_{0}(t, s) & =\operatorname{ch}[F(t, s)] K_{2}(s)-\operatorname{sh}[F(t, s)] K_{1}(s),
\end{aligned}
$$

with

$$
F(t, s)=\int_{s}^{t} g\left(t^{\prime}\right) d t^{\prime},
$$

where $\operatorname{ch}(x)$ and $\operatorname{sh}(x)$ denote the cosine and sine hyperbolic functions, respectively. $K_{1}(s)$ and $K_{2}(s)$ are arbitrary functions independent of time $t$, which are calculated using the boundary conditions; see Eq. (A3) and Eq. (A4). We take the limit $\gamma \ll \omega_{0}$ and write the propagator in a more convenient form,

$$
\begin{aligned}
D_{R}(t, s)= & (\operatorname{ch}[F(t, s)] \sin [\Omega(t-s)-\phi(s)] \\
& -\operatorname{sh}[F(t, s)] \cos [\Omega(t+s)+\phi(s)]) A(s) e^{-\gamma(t-s)},
\end{aligned}
$$


where we have replaced the functions $\mathrm{K} 1(\mathrm{~s})$ and $\mathrm{K} 2(\mathrm{~s})$ with two other unknown functions, $A(s)$ and $\phi(s)$, defined as

$$
\begin{aligned}
& K_{1}(s)=-A(s) \sin (\Omega s+\phi(s)) e^{\gamma s}, \\
& K_{2}(s)=A(s) \cos (\Omega s+\phi(s)) e^{\gamma s} .
\end{aligned}
$$

The functions $A(s)$ and $\phi(s)$ can be evaluated using the boundary conditions. We use the continuity condition, Eq. (A3), of the retarded Green's function,

$$
\sin [-\phi(t)] A(t)=0,
$$

together with the jump condition, Eq. (A3), at time $t \neq 0$,

$$
A(t) X(\Omega-\delta(t) \cos (2 \Omega t))=-2 \omega_{0},
$$

and find that

$$
\begin{aligned}
& \phi(s)=0, \\
& A(s)=-2,
\end{aligned}
$$

which gives, for the retarded Green's function,

$$
\begin{aligned}
D_{R}(t, s)= & -2 \theta(t-s)(\operatorname{ch}[F(t, s)] \sin [\Omega(t-s)] \\
& -\operatorname{sh}[F(t, s)] \cos [\Omega(t+s)]) e^{-\gamma(t-s)} .
\end{aligned}
$$

In the limit of a delta-shaped pump pulse $g(t)=r \delta(t)$, we have that

$$
\begin{aligned}
& F(t, s)=r \int_{s}^{t} \delta\left(t^{\prime}\right) d t^{\prime}=r, \quad \operatorname{sign}(t) \neq \operatorname{sign}(s), \\
& F(t, s)=r \int_{s}^{t} \delta\left(t^{\prime}\right) d t^{\prime}=0, \quad \operatorname{sign}(t)=\operatorname{sign}(s),
\end{aligned}
$$

with $\operatorname{sign}(t)$ the sign function. Thus, the retarded phonon Green's function is defined piece-wise and reads

$$
D_{R}(t, s)= \begin{cases}D_{\mathrm{eq}}^{R}(t, s), & \operatorname{sign}(t)=\operatorname{sign}(s), \\ D_{\mathrm{sq}}^{R}(t, s), & \operatorname{sign}(t) \neq \operatorname{sign}(s),\end{cases}
$$

with

$$
\begin{aligned}
D_{\mathrm{eq}}^{R}(t, s)= & -2 \theta(t-s) e^{-\gamma(t-s)} \sin [\Omega(t-s)], \\
D_{\mathrm{sq}}^{R}(t, s)= & -2 e^{-\gamma(t-s)} \theta(t-s)(\operatorname{ch}(r) \sin [\Omega(t-s)] \\
& -\operatorname{sh}(r) \cos [\Omega(t+s)]),
\end{aligned}
$$

where $D_{\mathrm{eq}}^{R}(t, s)$ and $D_{\mathrm{sq}}^{R}(t, s)$ stand for the equilibrium and squeezed retarded Green's function. The fact that the squeezed propagator is not a function of the time difference $D_{\mathrm{sq}}^{R}(t, s) \neq$ $D_{\mathrm{sq}}^{R}(t-s)$ is a consequence of breaking the time translational symmetry.

We now evaluate the function $\Delta_{R}(t, \omega)$ discussed in the text and defined as

$$
\Delta_{R}(t, \omega)=\int \Delta_{R}(t, t-\tau) e^{i \omega \tau} d \tau .
$$

In the time domain, the phonon propagators $D_{R}\left(t_{1}, t_{2}\right)$ of the squeezed phonon are defined piecewise [see Eq. (A20)]. Thus, we split the integral, Eq. (A21), over the two time domains and write

$$
\begin{aligned}
\Delta_{R}(t, \omega)= & \int_{-\infty}^{\infty} D_{\mathrm{eq}}^{R}(t, t-\tau) e^{i \omega \tau} d \tau \\
& +\int_{t}^{\infty}\left(D_{\mathrm{sq}}^{R}(t, t-\tau)-D_{\mathrm{eq}}^{R}(t, t-\tau)\right) e^{i \omega \tau} d \tau,
\end{aligned}
$$

where we have added and subtracted $\int_{t}^{\infty} D_{\mathrm{eq}}^{R}(t, t-\tau) e^{i \omega \tau} d \tau$ to extend the limits of the first integral to $+\infty$. The first term of the integral is the equilibrium contribution and is time independent. The second term is the out-of-equilibrium contribution to the retarded propagator $\Delta_{R}(t, \omega)$ and vanishes for a vanishing squeezing parameter $r=0$ or for negative times $t<0$. For positive times after the pump pulse $t>0$, we have

$$
\begin{aligned}
I(t, \omega) \equiv & \int_{t}^{\infty}\left(D_{\mathrm{sq}}^{R}(t, t-\tau)-D_{\mathrm{eq}}^{R}(\tau)\right) e^{i \omega \tau} d \tau \\
= & -2 \int_{t}^{\infty}\left(g_{2}(t) \sin (\Omega \tau)-g_{1}(t) \cos (\Omega \tau)\right) \\
& \times e^{(i \omega-\gamma) \tau} d \tau
\end{aligned}
$$

where the complex functions $g_{1}(t)$ and $g_{2}(t)$ are defined as

$$
\begin{aligned}
& g_{1}(t)=\operatorname{sh}(r) \cos (2 \Omega t) \\
& g_{2}(t)=\operatorname{ch}(r)-\operatorname{sh}(r) \sin (2 \Omega t)-1
\end{aligned}
$$

with $\Omega=\sqrt{\omega_{0}^{2}-\gamma^{2}}$. We define the complex function $A(t)=$ $g_{1}(t)+i g_{2}(t)$ and express the nonequilibrium part of the retarded Green's function $I(\omega, t)$ in a compact exponential form,

$$
\begin{aligned}
I(t, \omega)= & \int_{t}^{\infty}\left(A(t) e^{i \Omega \tau}+\bar{A}(t) e^{-i \Omega \tau}\right) e^{(i \omega-\gamma) \tau} d \tau \\
= & \frac{i}{2 \Omega}\left(A(t)(\omega-\Omega+i \gamma) e^{i(\omega+\Omega+i \gamma) t}\right. \\
& \left.+\bar{A}(t)(\omega+\Omega+i \gamma) e^{i(\omega-\Omega+i \gamma) t}\right) D_{\mathrm{eq}}^{R}(\omega),
\end{aligned}
$$

where $D_{\mathrm{eq}}^{R}(\omega)$ denotes the equilibrium retarded Green's function. Henceforth, we take the limit $\gamma \ll \omega_{0}$ for which $\Omega \approx \omega_{0}$, and we write the function $\Delta_{R}(t, \omega)$ as

$$
\Delta_{R}(t, \omega)=\left(1+\frac{i}{2 \omega_{0}} \theta(t) e^{-\gamma t} K(t, \omega) e^{i \omega t}\right) D_{\mathrm{eq}}^{R}(\omega)
$$

so that

$$
\begin{aligned}
D_{\mathrm{eq}}^{R}(\omega)= & \frac{2 \omega_{0}}{\omega^{2}+2 i \gamma \omega-\omega_{0}^{2}}, \\
K(t, \omega)= & A(t)\left(\omega-\omega_{0}+i \gamma\right) e^{i \omega_{0} t} \\
& +\bar{A}(t)\left(\omega+\omega_{0}+i \gamma\right) e^{-i \omega_{0} t} .
\end{aligned}
$$

This gives Eq. (4) in the text. The out-of-equilibrium part of the retarded Green's function, as defined in Eq. (A21), has a $2 \omega_{0}$ oscillatory component. This follows from the definition of the function $A(t)$, whose real and imaginary parts oscillate as $\cos \left(2 \omega_{0} t\right)$ and $\sin \left(2 \omega_{0} t\right)$, respectively.

We insert the Fourier transform of the retarded Green's function $\Delta_{R}(t, \omega)$ in the expression of the equal-time 
correlation function and write for time $t>0$

$$
\begin{aligned}
\left\langle X_{\mathrm{cl}}^{2}(t)\right\rangle & =\frac{i}{2 \pi} \int_{-\infty}^{+\infty} \Delta_{R}(t, \omega) D^{R}(t,-\omega) \Pi^{K}(\omega) d \omega \\
& =\frac{1}{2 \pi} \int_{-\infty}^{+\infty}(1-y(\omega, t)) D_{\mathrm{eq}}^{K}(\omega) d \omega,
\end{aligned}
$$

where $y(\omega, t)$ is the nonequilibrium part of the Keldysh Green's function and is given by

$$
\begin{aligned}
y(\omega, t) \equiv & \frac{1}{4 \omega_{0}^{2}} e^{-2 \gamma t} K(t, \omega) K(t,-\omega) \\
& -\frac{i}{2 \omega_{0}} e^{-\gamma t}\left(K(t,-\omega) e^{-i \omega t}+K(t, \omega) e^{i \omega t}\right) .
\end{aligned}
$$

We recall that the equilibrium Keldysh Green's function is given by

$$
\begin{aligned}
D_{\mathrm{eq}}^{K}(\omega) & =\frac{8 \gamma \omega_{0} \omega \operatorname{coth}[\omega / 2 T]}{\left(\left[\omega-\omega_{0}\right]^{2}+\gamma^{2}\right)\left(\left[\omega+\omega_{0}\right]^{2}+\gamma^{2}\right)} \\
& =\frac{2 \gamma \operatorname{coth}[\omega / 2 T]}{\left(\omega-\omega_{0}\right)^{2}+\gamma^{2}}-\frac{2 \gamma \operatorname{coth}[\omega / 2 T]}{\left(\omega+\omega_{0}\right)^{2}+\gamma^{2}},
\end{aligned}
$$

where we see that it is peaked at $\omega= \pm \omega_{0}$ with a width $\gamma$. Therefore, in the limit where $\gamma \ll \omega_{0}$, the $\operatorname{coth}[\omega / 2 T]$ is a slow function of the frequency and the Keldysh Green's function can be approximated by

$$
D_{\mathrm{eq}}^{K}(\omega) \approx \frac{2 \gamma \operatorname{coth}\left[\omega_{0} / 2 T\right]}{\left(\omega-\omega_{0}\right)^{2}+\gamma^{2}}+\frac{2 \gamma \operatorname{coth}\left[\omega_{0} / 2 T\right]}{\left(\omega+\omega_{0}\right)^{2}+\gamma^{2}}
$$

where we have used coth $\left[-\omega_{0} / 2 T\right]=-\operatorname{coth}\left[\omega_{0} / 2 T\right]$. Using the above three equations we obtain

$$
\begin{aligned}
\left\langle X_{\mathrm{cl}}^{2}(t)\right\rangle= & \frac{2}{\pi} \operatorname{coth}\left[\omega_{0} / 2 T\right] \int_{-\infty}^{+\infty} \frac{\gamma}{\left(\omega-\omega_{0}\right)^{2}+\gamma^{2}} \\
& \times\left[1-\frac{1}{4 \omega_{0}^{2}} e^{-2 \gamma t} K(t, \omega) K(t,-\omega)\right. \\
& \left.+\frac{i}{2 \omega_{0}} e^{-\gamma t}\left(K(t,-\omega) e^{-i \omega t}+K(t, \omega) e^{i \omega t}\right)\right] d \omega .
\end{aligned}
$$

We integrate over the frequency $\omega$ and obtain for the equaltime correlation function

$$
\begin{aligned}
\left\langle X_{c l}^{2}(t)\right\rangle= & 2 \operatorname{coth}\left[\omega_{0} / 2 T\right]\left[1-\frac{1}{4 \omega_{0}^{2}} e^{-2 \gamma t} K\left(t, \omega_{0}+i \gamma\right) K\left(t,-\omega_{0}-i \gamma\right)\right. \\
& \left.+\frac{i}{2 \omega_{0}} e^{-2 \gamma t}\left(K\left(t,-\omega_{0}-i \gamma\right) e^{-i \omega_{0} t}+K\left(t, \omega_{0}+i \gamma\right) e^{i \omega_{0} t}\right)\right] .
\end{aligned}
$$

We take the limit $\gamma \ll \omega_{0}$ and write the correlation function as

$$
\begin{aligned}
\left\langle X_{\mathrm{cl}}^{2}(t)\right\rangle= & 2 \operatorname{coth}\left[\omega_{0} / 2 T\right]\left[1+e^{-2 \gamma t} \bar{A}(t) A(t)\right. \\
& \left.+i e^{-2 \gamma t}(\bar{A}(t)-A(t))\right] .
\end{aligned}
$$

We replace the complex function $A(t)$ with its expression in Eq. (A24) and obtain for the variance after simplification

$$
\begin{aligned}
\Delta^{2} X(t)= & \operatorname{coth}\left[\omega_{0} / 2 T\right]\left[1+e^{-2 \gamma t}(\operatorname{ch}(2 r)-1)\right. \\
& \left.-\operatorname{sh}(2 r) \sin \left(2 \omega_{0} t\right) e^{-2 \gamma t}\right],
\end{aligned}
$$

where $r$ is the squeezing parameter. The variance of the atomic displacement oscillates at twice the frequency of the mode $2 \omega_{0}$. This is Eq. (5) in the text.

\section{APPENDIX B: TIME-RESOLVED SPECTRAL FUNCTION}

Here, we discuss the calculation details of the timeresolved spectral function defined as

$$
A(t, \omega) \equiv \operatorname{Im} D^{R}(t, \omega),
$$

where

$$
D^{R}(t, \omega)=\int D^{R}(t+\tau / 2, t-\tau / 2) e^{i \omega \tau} d \tau
$$

is the Wigner transform of the retarded Green's function. We derive the expression of $A(t, \omega)$ in the two scenarios discussed in the text, namely, a squeezed phonon and a phonon, with a cubic anharmonic potential, excited coherently by the pump. We show that the time-resolved spectral function is qualitatively different for these two cases and can therefore be used to detect phonon squeezed states.

\section{Case (i)}

Let us first discuss the signature of a squeezed phonon [case (i) in the text] on the spectral function $A(t, \omega)$. The out-of-equilibrium part of the Wigner transform is defined as

$$
\begin{aligned}
\Delta D^{R}(t, \omega) & \equiv D^{R}(t, \omega)-D_{\mathrm{eq}}^{R}(\omega) \\
& =\int_{-\infty}^{+\infty}\left(D^{R}(t+\tau / 2, t-\tau / 2)-D_{\mathrm{eq}}^{R}(\tau)\right) e^{i \omega \tau} d \tau,
\end{aligned}
$$

where $D_{\mathrm{eq}}^{R}(\omega)$ is the equilibrium retarded Green's function. At equilibrium, the Wigner transform coincides with its Fourier transform, hence, the retarded Green's function $D^{R}(t+\tau / 2, t-\tau / 2)$ of a squeezed phonon is different from equilibrium only for times where $\operatorname{Sign}(t+\tau / 2) \neq \operatorname{Sign}(t-$ $\tau / 2$ ). Therefore, the integral in Eq. (B3) is nonvanishing only 
for time $\tau>2 t$, where it can be written as

$$
\begin{aligned}
\Delta D^{R}(t, \omega)= & \int_{2 t}^{\infty}\left[D_{\mathrm{sq}}^{R}\left(t+\frac{\tau}{2}, t-\frac{\tau}{2}\right)-D_{\mathrm{eq}}^{R}(\tau)\right] e^{i \omega \tau} d \tau \\
= & -2(\operatorname{ch}(r)-1) \int_{2 t}^{\infty} \sin \left(\omega_{0} \tau\right) e^{-\gamma \tau} e^{i \omega \tau} d \tau \\
& +2 \operatorname{sh}(r) \cos \left(2 \omega_{0} t\right) \int_{2 t}^{\infty} e^{-\gamma \tau} e^{i \omega \tau} d \tau
\end{aligned}
$$

We get

$$
\begin{aligned}
\Delta D^{R}(t, \omega)= & +2 \operatorname{sh}(r) \cos \left(2 \omega_{0} t\right) \int_{2 t}^{\infty} e^{-\gamma \tau} e^{i \omega \tau} d \tau \\
& +i(\operatorname{ch}(r)-1) \int_{2 t}^{\infty} \\
& \times\left[e^{i\left(\omega+\omega_{0}+i \gamma\right) \tau}-e^{i\left(\omega-\omega_{0}+i \gamma\right) \tau}\right] d \tau
\end{aligned}
$$

We integrate over time $\tau$ and find

$$
\Delta D^{R}(t, \omega)=(1-\operatorname{ch}(r)) e^{-2 \gamma t}\left[\frac{e^{2 i\left(\omega+\omega_{0}\right) t}}{\omega+\omega_{0}+i \gamma}-\frac{e^{2 i\left(\omega-\omega_{0}\right) t}}{\omega-\omega_{0}+i \gamma}\right]+2 i \operatorname{sh}(r) \cos \left(2 \omega_{0} t\right) e^{-2 \gamma t} \frac{e^{2 i \omega t}}{\omega+i \gamma}
$$

Thus the spectral function $A\left(t, \omega_{0}\right)$ evaluated at frequency $\omega_{0}$ has a $4 \omega_{0}$ oscillatory component in the time domain, coming from the first and third terms above.

\section{Case (iii)}

We now calculate the spectral function of a phonon, with a cubic anharmonic potential, excited coherently by the pump [case (iii) in the text). The first-order correction of the retarded Green's function, discussed in the text, is given by

$$
D_{R, 1}\left(t, t^{\prime}\right)=2 \int D_{R, \mathrm{eq}}(t-s) D_{R, \mathrm{eq}}\left(t^{\prime}-s\right) u(s) d s
$$

We Wigner transform the retarded Green's function and write

$$
\begin{aligned}
D_{R, 1}(t, \omega)= & 2 \int D_{R, \mathrm{eq}}(t+\tau / 2-s) \\
& \times D_{R, \mathrm{eq}}(t-\tau / 2-s) u(s) e^{i \omega \tau} d \tau d s .
\end{aligned}
$$

For simplicity, we discuss the case of an impulsive stimulation for which the retarded Green's function and the average atomic displacement $u(t)$ are given by

$$
\begin{aligned}
D_{R, \mathrm{eq}}\left(t, t^{\prime}\right) & =-2 \theta\left(t-t^{\prime}\right) \sin \left[\omega_{0}\left(t-t^{\prime}\right)\right] e^{-\gamma\left(t-t^{\prime}\right)}, \\
u(t) & =\theta(t) Q_{0} \sin \left(\omega_{0} t\right) e^{-\gamma t}=-\frac{Q_{0}}{2} D_{R, \mathrm{eq}}(t),
\end{aligned}
$$

where $Q_{0}$ denotes the amplitude of the coherent phonon oscillation. We Fourier transform and obtain

$$
\begin{aligned}
D_{R, 1}(t, \omega)= & \frac{Q_{0}}{2 \pi} \int D_{R, \text { eq }}(\Omega) D_{R, \text { eq }}(\omega-\Omega / 2) \\
& \times D_{R, \text { eq }}(\omega+\Omega / 2) e^{-i \Omega t} d \Omega .
\end{aligned}
$$

Finally, we integrate over frequency $\Omega$ and find that

$$
\begin{aligned}
D_{R, 1}(t, \omega)= & Q_{0} i e^{-\gamma t}\left[\frac{4 \omega_{0}^{2} e^{-i \omega_{0} t}}{A\left(\omega, \omega_{0}\right)}-\frac{4 \omega_{0}^{2} e^{i \omega_{0} t}}{A\left(\omega,-\omega_{0}\right)}\right] \\
& +8 Q_{0} i e^{-2 \gamma t}\left[\frac{4 \omega_{0}^{2} e^{2 i t\left(\omega+\omega_{0}\right)}}{B\left(\omega, \omega_{0}\right)}-\frac{4 \omega_{0}^{2} e^{2 i t\left(\omega-\omega_{0}\right)}}{B\left(\omega,-\omega_{0}\right)}\right]
\end{aligned}
$$

with

$$
\begin{aligned}
A\left(\omega, \omega_{0}\right)= & \left(\omega-\frac{3 \omega_{0}}{2}+i \frac{3 \gamma}{2}\right)\left(\omega+\frac{3 \omega_{0}}{2}+i \frac{\gamma}{2}\right) \\
& \times\left(\omega+\frac{\omega_{0}}{2}+i \frac{3 \gamma}{2}\right)\left(\omega-\frac{\omega_{0}}{2}+i \frac{\gamma}{2}\right), \\
B\left(\omega, \omega_{0}\right)= & \left(4 \omega+4 \omega_{0}+4 i \gamma\right)(4 \omega+4 i \gamma) \\
& \times\left(2 \omega+3 \omega_{0}+i \gamma\right)\left(2 \omega+\omega_{0}+i \gamma\right) .
\end{aligned}
$$

From the above expression, we see that the spectral function $A\left(t, \omega_{0}\right)$ evaluated at frequency $\omega_{0}$ has both a $4 \omega_{0}$ (third term above) and an $\omega_{0}$ oscillatory component (first and second terms above) in the time domain.

\section{APPENDIX C: SPECTRAL FUNCTION OUT OF EQUILIBRIUM}

In this Appendix, we show that the time-dependent spectral function can be extracted from the difference between the Stokes and the anti-Stokes Raman intensities. Following the theory of time- and frequency-resolved Raman spectroscopy, the scattering intensity $I(t, \omega) \propto D^{>}(t, \omega)$, where $D^{>}(t, \omega)$ is the Wigner transform of the two times greater function. The relevant references can be found in the text. Here, we derive a relation that connects the greater and retarded Green's function out of equilibrium.

The greater component of the Green's function satisfies the relation

$$
D^{>}\left(t, t^{\prime}\right)=\frac{1}{2}\left(D^{K}\left(t, t^{\prime}\right)+D^{R}\left(t, t^{\prime}\right)-D^{A}\left(t, t^{\prime}\right)\right)
$$

We use the linear property of the Wigner transformation and find that

$$
D_{W}^{>}(t, \omega)=\frac{1}{2}\left(D_{W}^{K}(t, \omega)+D_{W}^{R}(t, \omega)-D_{W}^{A}(t, \omega)\right) .
$$

The Keldysh Green's function is symmetric with respect to its two time arguments. Therefore, its Wigner transform satisfies the relation $D_{W}^{K}(t, \omega)=D_{W}^{K}(t,-\omega)$. Similarly, the advanced and retarded Green's function are related by the identity $D^{R}\left(t, t^{\prime}\right)=D^{A}\left(t^{\prime}, t\right)$, which implies that 
$D_{W}^{R}(t, \omega)=D_{W}^{A}(t,-\omega)$. Thus, we have that

$$
\begin{aligned}
D_{W}^{>}(t, \omega)-D_{W}^{>}(t,-\omega) & =\left(D_{W}^{R}(t, \omega)-D_{W}^{R}(t,-\omega)\right) \\
& =2 i \operatorname{Im}\left[D_{W}^{R}(t, \omega)\right],
\end{aligned}
$$

where we have used $D_{W}^{R}(t, \omega)=D_{W}^{R}(t,-\omega)^{*}$. Finally, we use the fact that the intensity of the Raman spectrum is propor- tional to the greater Green's function $I(t, \omega) \propto D^{>}(t, \omega)$ and find that

$$
A(t, \omega) \propto I(t, \omega)-I(t,-\omega) .
$$

The above relation shows that the out-of-equilibrium spectral function $A(t, \omega)$ can be measured using time-resolved Raman spectroscopy.
[1] For reviews see, e.g., J. Orenstein, Phys. Today 65, 44 (2012); J. Zhang and R. D. Averitt, Annu. Rev. Mater. Res. 44, 19 (2014); D. Nicoletti and A. Cavalleri, Adv. Opt. Photon. 3, 401 (2016); C. Giannetti, M. Capone, D. Fausti, M. Fabrizio, F. Parmigiani, and D. Mihailovic, Adv. Phys. 65, 58 (2016); D. N. Basov, R. D. Averitt, and D. Hsieh, Nat. Mater. 16, 1077 (2017).

[2] H. Okamoto, H. Matsuzaki, T. Wakabayashi, Y. Takahashi, and T. Hasegawa, Phys. Rev. Lett. 98, 037401 (2007).

[3] D. Fausti, R. I. Tobey, N. Dean, S. Kaiser, A. Dienst, M. C. Hoffmann, S. Pyon, T. Takayama, H. Takagi, and A. Cavalleri, Science 331, 189 (2011).

[4] M. Liu, H. Y. Hwang, H. Tao, A. C. Strikwerda, K. Fan, G. R. Keiser, A. J. Sternbach, K. G. West, S. Kittiwatanakul, J. Lu, S. A. Wolf, F. G. Omenetto, X. Zhang, K. A. Nelson, and R. D. Averitt, Nature 487, 345 (2012).

[5] A. Zong, P. E. Dolgirev, A. Kogar, E. Ergeçen, M. B. Yilmaz, Y.-Q. Bie, T. Rohwer, I-C. Tung, J. Straquadine, X. Wang, Y. Yang, X. Shen, R. Li, J. Yang, S. Park, M. C. Hoffmann, B. K. Ofori-Okai, M. E. Kozina, H. Wen, X. Wang, I. R. Fisher, P. Jarillo-Herrero, and N. Gedik, Phys. Rev. Lett. 123, 097601 (2019).

[6] M. Mitrano, A. Cantaluppi, D. Nicoletti, S. Kaiser, A. Perucchi, S. Lupi, P. Di Pietro, D. Pontiroli, M. Riccò, S. R. Clark, D. Jaksch, and A. Cavalleri, Nature 530, 461 (2016).

[7] A. Kogar, A. Zong, P. E. Dolgirev, X. Shen, J. Straquadine, Y.Q. Bie, X. Wang, T. Rohwer, I.-C. Tung, Y. Yang, R. Li, J. Yang, S. Weathersby, S. Park, M. E. Kozina, E. J. Sie, H. Wen, P. Jarillo-Herrero, I. R. Fisher, X. Wang, and N. Gedik, Nat. Phys. 16, 159 (2019).

[8] M. Buzzi, D. Nicoletti, M. Fechner, N. Tancogne-Dejean, M. A. Sentef, A. Georges, M. Dressel, A. Henderson, T. Siegrist, J. A. Schlueter, K. Miyagawa, K. Kanoda, M.-S. Nam, A. Ardavan, J. Coulthard, J. Tindall, F. Schlawin, D. Jaksch, and A. Cavalleri, Phys. Rev. X 10, 031028 (2020).

[9] J. Li, H. U. R. Strand, P. Werner, and M. Eckstein, Nat. Commun. 9, 4581 (2018).

[10] A. Matthies, J. Li, and M. Eckstein, Phys. Rev. B 98, 180502(R) (2018).

[11] S. De Silvestri, J. G. Fujimoto, E. P. Ippen, E. B. Gamble, L. R. Williams, and K. A. Nelson, Chem. Phys. Lett. 116, 146 (1985).

[12] Y.-X. Yan, E. B. Gamble, and K. A. Nelson, J. Chem. Phys. 83, 5391 (1985).

[13] T. K. Cheng, S. D. Brorson, A. S. Kazeroonian, J. S. Moodera, G. Dresselhaus, M. S. Dresselhaus, and E. P. Ippen, Appl. Phys. Lett. 57, 1004 (1990).

[14] H. J. Zeiger, J. Vidal, T. K. Cheng, E. P. Ippen, G. Dresselhaus, and M. S. Dresselhaus, Phys. Rev. B 45, 768 (1992).

[15] R. Merlin, Solid State Commun. 102, 207 (1997).
[16] For a review see, e.g., K. Ishioka and O. V. Misochko, Springer Ser. Chem. Phys. 98, 23 (2010).

[17] M. Först, C. Manzoni, S. Kaiser, Y. Tomioka, Y. Tokura, R. Merlin, and A. Cavalleri, Nat. Phys. 7, 854 (2011).

[18] A. Subedi, A. Cavalleri, and A. Georges, Phys. Rev. B 89, 220301(R) (2014).

[19] R. Mankowsky, A. Subedi, M. Först, S. O. Mariager, M. Chollet, H. T. Lemke, J. S. Robinson, J. M. Glownia, M. P. Minitti, A. Frano, M. Fechner, N. A. Spaldin, T. Loew, B. Keimer, A. Georges, and A. Cavalleri, Nature 516, 71 (2014).

[20] M. Lakehal and I. Paul, Phys. Rev. B 99, 035131 (2019).

[21] See, e.g., X. Hu and F. Nori, Phys. Rev. Lett. 76, 2294 (1996); Phys. Rev. B 53, 2419 (1996).

[22] P. D. Drumond and Z. Ficek (eds.), Quantum Squeezing. Springer Series on Atomic, Optical, and Plasma Physics (Springer-Verlag, Berlin, 2004).

[23] M. Knap, M. Babadi, G. Refael, I. Martin, and E. Demler, Phys. Rev. B 94, 214504 (2016).

[24] D. M. Kennes, E. Y. Wilner, D. R. Reichman, and A. J. Millis, Nat. Phys. 13, 479 (2017).

[25] E. E. Wollman, C. U. Lei, A. J. Weinstein, J. Suh, A. Kronwald, F. Marquardt, A. A. Clerk, and K. C. Schwab, Science 349, 952 (2015).

[26] A. A. Clerk, K. W. Lehnert, P. Bertet, and Y. Nakamura, Nat. Phys. 16, 257 (2020).

[27] G. A. Garrett, A. G. Rojo, A. K. Sood, J. F. Whitaker, and R. Merlin, Science 275, 1638 (1997).

[28] G. A. Garrett, J. F. Whitaker, A. K. Sood, and R. Merlin, Opt. Express 1, 387 (1997).

[29] A. Bartels, T. Dekorsy, and H. Kurz, Phys. Rev. Lett. 84, 2981 (2000).

[30] O. V. Misochko, K. Sakai, and S. Nakashima, Phys. Rev. B 61, 11225 (2000).

[31] S. L. Johnson, P. Beaud, E. Vorobeva, C. J. Milne, É. D. Murray, S. Fahy, and G. Ingold, Phys. Rev. Lett. 102, 175503 (2009).

[32] E. S. Zijlstra, L. E. Díaz-Sánchez, and M. E. Garcia, Phys. Rev. Lett. 104, 029601 (2010).

[33] M. Trigo, M. Fuchs, J. Chen, M. P. Jiang, M. Cammarata, S. Fahy, D. M. Fritz, K. Gaffney, S. Ghimire, A. Higginbotham, S. L. Johnson, M. E. Kozina, J. Larsson, H. Lemke, A. M. Lindenberg, G. Ndabashimiye, F. Quirin, K. SokolowskiTinten, C. Uher, G. Wang, J. S. Wark, D. Zhu, and D. A. Reis, Nat. Phys. 9, 790 (2013).

[34] M. Esposito, K. Titimbo, K. Zimmermann, F. Giusti, F. Randi, D. Boschetto, F. Parmigiani, R. Floreanini, F. Benatti, and D. Fausti, Nat. Comm. 6, 10249 (2015). 
[35] F. Benatti, M. Esposito, D. Fausti, R. Floreanini, K. Titimbo, and K. Zimmermann, New J. Phys. 19, 023032 (2017).

[36] A. Kamenev, Field Theory of Non-Equilibrium Systems (Cambridge University Press, Cambridge, UK, 2011).

[37] M. Schiró and K. Le Hur, Phys. Rev. B 89, 195127 (2014).

[38] Y. Wang, T. P. Devereaux, and C.-C. Chen, Phys. Rev. B 98, 245106 (2018).
[39] A. M. Shvaika, O. P. Matveev, T. P. Devereaux, and J. K. Freericks, Condens. Matter Phys. 21, 33707 (2018).

[40] G. Batignani, D. Bossini, N. Di Palo, C. Ferrante, E. Pontecorvo, G. Cerullo, A. Kimel, and T. Scopigno, Nat. Photon. 9, 506 (2015).

[41] K. E. Dorfman, B. P. Fingerhut, and S. Mukamel, J. Chem. Phys. 139, 124113 (2013). 